\title{
Collision Avoidance for Satellites in Formation Flight
}

\author{
G. L. Slater, ${ }^{*}$ S. M. Byram, ${ }^{\dagger}$ and T. W. Williams* \\ University of Cincinnati, Cincinnati, Ohio 45221-0070
}

\begin{abstract}
Satellites in a formation might need to maneuver to avoid potential collisions that may occur when foreign objects enter the formation, or when a satellite within the formation drifts into the path of another. In either case we seek to determine the probability of a future collision based on current state knowledge and the uncertain dynamic environment, and further to determine a control strategy to reduce the collision probability to an acceptable level while minimizing the $\Delta V$ required for the maneuver. The approach taken in this paper is to propagate the uncertainty covariance using linear theory and determine the probability that the relative displacement between two objects is less than some "collision metric." This probability will be a function of the initial conditions, the uncertainty in the initial state and disturbing acceleration, and the time-to-go before closest approach. The intent of the paper is to examine the evolution of this probability and to determine an effective maneuver algorithm that can minimize the probability of collision while reducing the energy expenditure in the maneuver. Numerical values are used for satellites in a tightly spaced, low-Earth-orbit formation. For satellites in a close formation, it is demonstrated that the uncertain disturbance environment can make efficient $\Delta V$ maneuvers difficult to determine, as collision probabilities can vary rapidly (on the orbital timescale.) Remarks and some sample calculations on the total $\Delta V$ required for an evasion maneuver are presented.
\end{abstract}

\section{Introduction}

$\mathbf{T}$ HE use of multiple spacecraft in a close formation can have numerous advantages over one single, larger spacecraft. The formation problem introduces new problems, however, in maintaining the formation geometry and in preventing inadvertent collisions between the spacecraft as a result of inaccuracy in knowledge of individual spacecraft state and/or possible failures of one of the spacecraft. The problem to be considered in this paper is specifically the collision probability for satellites in the formation and determination of spacecraft maneuvers to reduce the collision probability to an acceptable level of risk.

Although the probability of a spacecraft colliding with another space object has long been a subject of concern, recent work to look at multisatellite formations in close proximity has caused increased emphasis in this area. A set of benchmark formations is described in Carpenter et al. ${ }^{1}$ Some pertinent work on formation geometry and control has been done, for example, by Carpenter ${ }^{2}$ and Sabol et al. ${ }^{3}$ Calculation of collision probability requires integration of the probability density function over the three-dimensional volume (or perhaps two dimensions) of possible spacecraft locations (for example, see Chan, ${ }^{4}$ Alfriend and coauthors, ${ }^{5}$ Patera,${ }^{6}$ and Patera and Peterson ${ }^{7}$.

In this study we examine collision probability as it is related to the accuracy of orbital navigation and to the disturbance environment. Where and how to apply velocity corrections to avoid potential collisions (or "near approaches") based on these assumptions is the goal of this work. For this study we will examine specifically the case of vehicles in a low Earth nominal circular orbit formation. In the benchmark formation, ${ }^{1}$ six satellites describe a circular ground track pattern of $500 \mathrm{~m}$ diam in a $400-\mathrm{km}$-altitude circular orbit. The formation is defined by a reference orbit, which is (near) circular and

Presented as Paper 2004-5216 at the AIAA/AAS Astrodynamics Specialists Conference, Providence, RI, 15 August 2004; received 24 March 2005; revision received 7 July 2005; accepted for publication 2 August 2005. Copyright (C) 2005 by G. L. Slater. Published by the American Institute of Aeronautics and Astronautics, Inc., with permission. Copies of this paper may be made for personal or internal use, on condition that the copier pay the $\$ 10.00$ per-copy fee to the Copyright Clearance Center, Inc., 222 Rosewood Drive, Danvers, MA 01923; include the code 0731-5090/06 \$10.00 in correspondence with the CCC.

*Professor, Department of Aerospace Engineering and Engineering Mechanics. Associate Fellow AIAA

$\dagger$ Graduate Research Assistant; currently Graduate Research Assistant, Department of Aerospace Engineering, University of Michigan, Ann Arbor, Michigan. Student Member AIAA. sun synchronous. The six satellites are equally spaced in two sets of three satellites, with the two sets forming oppositely inclined planes such that the projected motion is a 500-m-diam circle when projected into the along-track/cross-track plane. For the formation to continue in a stable formation, the orbital semimajor axes of all satellites must be the same. Viewed from the linearized equations, the radial and along-track perturbations must be related so as to eliminate the secular terms that appear in the solution to the linearized equations.

Collisions can arise either from a foreign object passing through the formation, or from lack of control of one or more of the formation satellites such that two satellites might drift toward each other. The former problem is in line with classical collision analysis of spacecraft debris, whereas the latter is more indicative of a problem peculiar to formation flight. The main differences between these two cases as we perceive them are that 1) the relative velocities of the two objects will generally be small with formation objects and that 2) for formation flight the same linearized model is relevant for the two objects being considered. In this paper we will not deal specifically with determining the specific navigation method or accuracy or the quantitative specification of the disturbance environment.

The outline of this paper is as follows. In Sec. II we discuss the basic probabilistic model used in calculation of collision probabilities and ultimately to determine appropriate velocity corrections. Section III refines this analysis to determine the actual collision probability based on available state and disturbance information. Velocity correction requirements to avoid collisions are discussed in Sec. IV, followed by conclusions.

\section{Probabilistic Model}

For this problem we consider the question of determining the future most likely location of an individual satellite in a low-Earthorbit formation and ultimately determine the probability that two or more satellites in the formation might "collide" (i.e., approach closer than an allowable conflict radius). In this paper we will assume we can look at the motion of an individual satellite linearized about a nominal two-body circular orbit that defines the centroid of the formation. The linearized equations are the standard ClohessyWiltshire (or CW) equations ${ }^{8}$ (also referred to as the Hill's equations), which we write in normalized form as

$$
\dot{\boldsymbol{X}}=A \boldsymbol{X}+B \boldsymbol{w}+\Gamma \boldsymbol{u}
$$

where the state vector has components

$$
\boldsymbol{X}=\left[\begin{array}{llllll}
x & z & v_{x} & v_{z} & y & v_{y}
\end{array}\right]^{T}
$$

with $x$ the coordinate in the direction of the reference velocity, $z$ 


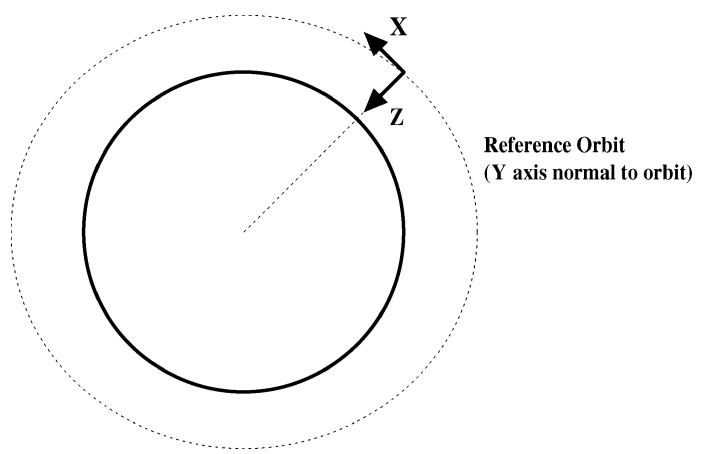

Fig. 1 Reference frame used to study the linearized motions about a nominal circular path.

of the orbit normal (such that $x, y, z$ form a right-handed triad) (see Fig. 1). (These coordinates are equivalent to the standard set used in spacecraft and aircraft attitude control. In the collision literature the $x$ axis is sometimes referred to as the VBAR direction, whereas the $z$ axis is the RBAR direction.) Here $\boldsymbol{w}$ is a disturbance vector, and $\boldsymbol{u}$ is a vector of exogenous control forces. In normalized units the system $A$ matrix is given by

$$
A=\left[\begin{array}{rrrrrr}
0 & 0 & 1 & 0 & 0 & 0 \\
0 & 0 & 0 & 1 & 0 & 0 \\
0 & 0 & 0 & 2 & 0 & 0 \\
0 & 3 & -2 & 0 & 0 & 0 \\
0 & 0 & 0 & 0 & 0 & 1 \\
0 & 0 & 0 & 0 & -1 & 0
\end{array}\right]
$$

$B$ and $\Gamma$ are identical and have the form

$$
B=\Gamma=\left[\begin{array}{lll}
0 & 0 & 0 \\
0 & 0 & 0 \\
1 & 0 & 0 \\
0 & 1 & 0 \\
0 & 0 & 0 \\
0 & 0 & 1
\end{array}\right]
$$

The normalized coordinates correspond to a unit distance length corresponding to the nominal orbit radius and a time unit such that the nominal circular orbit speed is unity (hence the orbital period is $P=2 \pi$ time units).

Note that the in-plane motion is decoupled from motion normal to the orbit so that we can generally decompose this sixth-order system into a fourth-order problem for in-plane motion and a second-order problem for the motion normal to the nominal orbit plane. In general we will assume this separation holds true even with control and disturbances. It is certainly possible that state-dependent disturbance or control can in fact couple the in-plane and out-of-plane motions, but such coupling will not explicitly be considered in this paper. As a remark we note that the specific linear model defined by Eqs. (3) and (4) is for the standard two-body case. Additional terms could be included in the linearized model (such as gravity harmonics), as well as noncircular reference orbits, ${ }^{9}$ and in that case most calculations must be performed numerically.

The fundamental matrix of Eq. (3) is of interest in our probability calculations and is easily computed analytically. Restricting to the in-plane case (i.e., dropping the last two rows and columns of $A$ ), it can be shown that the solution is
Table 1 Table of probabilities associated with equiprobability ellipsoids of $k$ standard deviations in a space of $\boldsymbol{n}$ dimensions

\begin{tabular}{lccc}
\hline \hline$n / k$ & 1 & 2 & 3 \\
\hline 1 & 0.683 & 0.955 & 0.997 \\
2 & 0.394 & 0.865 & 0.989 \\
3 & 0.200 & 0.739 & 0.971 \\
\hline \hline
\end{tabular}

We are interested in the propagation of uncertainties in both initial conditions, disturbances and control. Consider initially the problem of uncertainties in initial state and the effect of uncertainties in the dynamic model as reflected in the disturbance input $\boldsymbol{w}$. (i.e., neglect for now any effect of the control $\boldsymbol{u}$.) We assume that $\boldsymbol{w}=N(0, W)$ where the notation $N(\overline{\boldsymbol{w}}, W)$ implies the variable $\boldsymbol{w}$ has a "normal" or Gaussian distribution with mean $\bar{w}$ and covariance $W$. Similarly assume that $X(0)=N\left(0, P_{0}\right)$. Then it is well known that $X(t)=N[0, P(t)]$ for all time where

$$
\dot{P}=A P+P A^{T}+B W B^{T}
$$

The solution for $P(t)$ can be obtained analytically for the circular reference orbital model. In fact, an explicit solution for $P(t)$ can be written in terms of the matrix exponential (5) as

$$
P(t)=\Phi\left(t, t_{0}\right) P_{0} \Phi^{T}\left(t, t_{0}\right)+\int_{t_{0}}^{t} \Phi(t, \tau) B W B^{T} \Phi^{T}(t, \tau) \mathrm{d} \tau
$$

Assume for now we have generated a $P$ matrix for some specified time. Then (assuming $X$ is zero mean) the probability density function for $X$ is given by

$$
p d f(\boldsymbol{X})=\left(1 / \sqrt[n]{2 \pi}|P|^{\frac{1}{2}}\right) \exp \left(-\frac{1}{2} \boldsymbol{X}^{T} P^{-1} \boldsymbol{X}\right)
$$

The covariance matrix $P(t)$ determines the likelihood that the state vector is contained within an ellipsoid of probability. Although the $P$ matrix represents the entire state, we are only interested in the position covariances in terms of determining collision probabilities. Assume then we partition $P$ to take the partition $P_{11}$ corresponding to the position states (either two or three states depending on whether we are considering the two or three dimensional motion of the satellites) The eigenvalues and eigenvectors of $P_{11}$ determine the principle directions and the magnitude of the covariances along those directions (for example, see Bryson and Ho, ${ }^{10}$ Chapter 10). The probabilities of the actual $n$-dimension position vector lying within an ellipsoid with major axes of $k$ standard deviation units are given by Bryson and $\mathrm{Ho}^{10}$ as shown in Table 1 .

Thus in two dimensions, the probability of lying within a $3 \sigma$ ellipsoid is 0.989. A typical curve showing the evolution of the ellipsoid of probability can be seen on Fig. 2. This case corresponds to zero initial state uncertainty and to unit acceleration uncertainty in the $x$ (along-track) direction. Because the covariance equation is linear, it is instructive to create unit solutions with initial uncertainty along one state component with all other terms zero. In this case each individual $P(0)$ will be rank one, and hence the resulting $P(t)$ will also be unity rank. These unit rank solutions will have a single positive eigenvalue with the remaining eigenvalues all zero. The eigenvector of the $i$ th solution is $\boldsymbol{e}_{i}$, the $i$ th column of $\Phi(t)$, and the associated eigenvalue is $\lambda=\left|\boldsymbol{e}_{i}\right|^{2}$. Thus by inspection of the fundamental matrix we see that uncertainties in the radial $(z)$ direction will cause the along-track $(x)$ uncertainty to grow linearly with time as $6 t$ with a small oscillation superposed, whereas the radial uncertainty has only a periodic variation. Similarly, an initial $x$-velocity uncertainty causes a secular growth in uncertainty along

$$
\Phi(t, \tau)=\left[\begin{array}{cccc}
1 & 6(t-\tau)-6 \sin (t-\tau) & -3(t-\tau)+4 \sin (t-\tau) & -2 \cos (t-\tau)+2 \\
0 & 4-3 \cos (t-\tau) & 2 \cos (t-\tau)-2 & \sin (t-\tau) \\
0 & -6 \cos (t-\tau)+6 & -3+4 \cos (t-\tau) & 2 \sin (t-\tau) \\
0 & 3 \sin (t-\tau) & -2 \sin (t-\tau) & \cos (t-\tau)
\end{array}\right]
$$




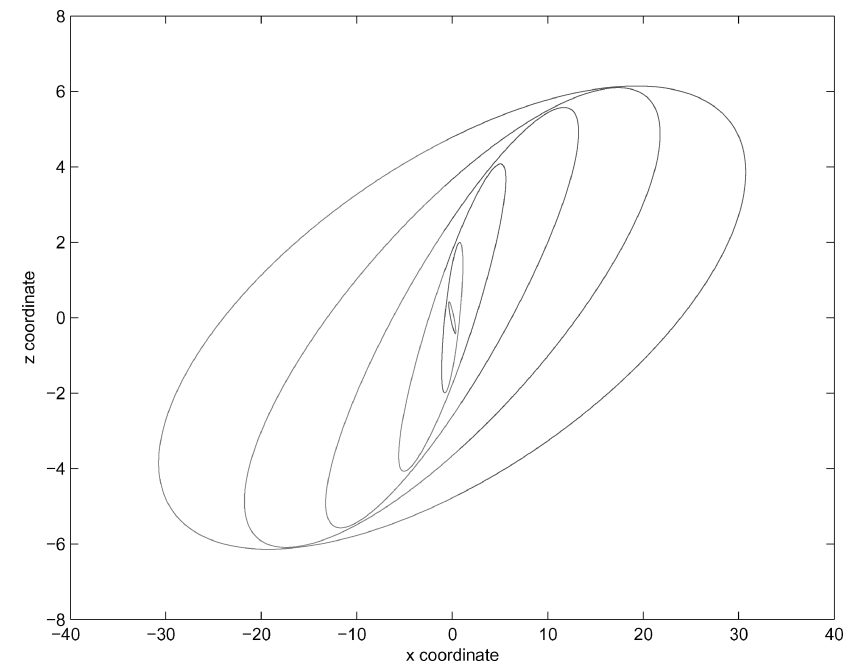

Fig. 2 Evolution of position probability ellipsoid (normalized units) caused by a unity along-track random acceleration. Ellipsoids drawn at integer times from 1 to 6 , where the orbit period is $2 \pi$.

track, while the $z$-velocity term causes only periodic uncertainty. Uncertainties in disturbing acceleration along the trajectory must be integrated as in Eq. (7). An acceleration component along only a single axis will produce a position covariance, which grows as the integral of $\boldsymbol{e}_{i} \boldsymbol{e}_{i}^{T}$. Thus for along-track accelerations, the position covariance grows as $t^{3}$ (thus the standard deviation of position caused by this acceleration grows as $t^{1.5}$ ). An acceleration in the normal direction cause a covariance growth only proportional to $t$. Of course in the general case, uncertainties of all types exist, and so the covariance will be full rank and have significant variability with time. The secular terms dominate the solution quickly however, but they do not necessarily dominate the collision probability as will be discussed shortly.

\section{A. Physical Units Description}

The preceding figure was shown using the normalized units previously defined. Consider then the mapping from these units to a nominal formation linearized about the reference condition:

$$
\begin{gathered}
a=1 \text { Distance Unit }=6.778 \cdot 10^{6} \mathrm{~m} \\
\text { Orbit period }=5535 \mathrm{~s} \\
\text { or } \quad 1 \text { Time Unit }=\frac{5535}{2 \pi}=8.8386 \cdot 10^{2} \mathrm{~s}
\end{gathered}
$$

Thus for example we can compute in the normalized units, and because the model is linear,we can convert to arbitrary physical units by creating appropriate conversion factors. Using the preceding condition, we can compute

$$
\begin{gathered}
1 D U=6.778 \cdot 10^{6} \mathrm{~m} \\
1 D U / T U=7.6686 \cdot 10^{3} \mathrm{~m} / \mathrm{s} \\
1 D U / T U^{2}=8.6763 \mathrm{~m} / \mathrm{s}^{2}
\end{gathered}
$$

For example, assume we have generated the covariance matrix as a result of unit uncertainty $(1 D U)$ in initial position. Because we are looking at position errors translating into position errors, the scale factors are equal, and we can assume whatever physical units for the initial covariance are the same units as for the final covariance. A covariance computed as a result of initial velocity uncertainty of $1 D U / T U$ and plotted in $D U$ can be converted to physical units by multiplying by the appropriate scale factors. For example, an uncertainty of $1 \mathrm{~cm} / \mathrm{s}$ corresponds to a velocity uncertainty of $1.3040 \cdot 10^{-6} \mathrm{DU} / \mathrm{TU}\left(10^{-2} / 7668.6\right)$. To convert the distance to meters multiply by the $D U$ to meter conversion above. Thus using the unit solution for velocity uncertainty, the physical uncertainty in meters caused by a $1-\mathrm{cm} / \mathrm{s}$ velocity uncertainty is obtained by multiplying the distance unit curve by $8.839\left(6.778 \cdot 10^{6} * 1.3040 \cdot 10^{-6}\right)$. We use the same logic for acceleration uncertainties, with one important distinction. If we timescale the equations of motion (as we did in introducing our normalized time unit), the covariance solution is not preserved. The explanation for this is buried in the mathematical subtleties (and difficulties) of white-noise driving ordinary differential equations. To preserve covariances in a timescale transformation, we must multiply the disturbance covariance matrix $W$ in Eqs. (6) and (7) by the timescale factor in addition to any other unit conversion factors. The following equations can be used to convert the normalized to various physical units for the benchmark problem.

Uncertainty units $\rightarrow$ multiply by for meters position displacement

$$
\begin{aligned}
1 \mathrm{~m} & \rightarrow 1 \\
1 \mathrm{~cm} / \mathrm{s} & \rightarrow 8.839 \\
1 \mathrm{~cm} / \mathrm{s}^{2} & \rightarrow 1.0152 \cdot 10^{4}
\end{aligned}
$$

\section{B. Relative Motion Between Two Satellites}

The prior derivation can be used to compute the probability that a satellite lies within a region in space (defined relative to the reference orbit in the linearization process). In our case we are interested in the probability that two or more spacecraft in close formation might violate some minimum separation, or what we will say is the probability that the two vehicles will "collide." Consider we have two satellites with state vector $\boldsymbol{X}_{\boldsymbol{1}}$ and $\boldsymbol{X}_{2}$ respectively, and where each individual motion can be modeled by the linearized model (1). The relative state defined by

$$
\delta X=X_{2}-X_{1}
$$

also clearly satisfies Eq. (1) with the addition that the control and disturbance terms are the difference of the terms in Eq. (1). In general, the initial relative covariances will not be the sum of the two terms because of correlations but can be computed as

$$
\begin{aligned}
& E\left\{[\delta \boldsymbol{X}(0)-\overline{\boldsymbol{\delta} \boldsymbol{X}}(0)][\delta \boldsymbol{X}(0)-\overline{\boldsymbol{\delta} \boldsymbol{X}}(0)]^{T}\right\}=P_{1}(0)+P_{2}(0) \\
& -E\left\{\left[\boldsymbol{X}_{1}(0)-\overline{\boldsymbol{X}}_{1}(0)\right]\left[\boldsymbol{X}_{2}(0)-\overline{\boldsymbol{X}}_{2}(0)\right]^{T}\right\} \\
& -E\left\{\left[\boldsymbol{X}_{2}(0)-\overline{\boldsymbol{X}}_{2}(0)\right]\left[\boldsymbol{X}_{1}(0)-\overline{\boldsymbol{X}}_{1}(0)\right]^{T}\right\}
\end{aligned}
$$

where $P_{1}(0)$ and $P_{2}(0)$ are the initial covariances of $\boldsymbol{X}_{1}$ and $\boldsymbol{X}_{2}$, respectively. Similarly disturbing accelerations are likely to be correlated on two close proximity spacecraft, as for example, as a result of drag. Discussion of the exact nature of some disturbances is discussed in Williams et al. ${ }^{11}$ For the current work we will assume some appropriate model has been created to model these relative uncertainties. Hence, the covariance equation for the state difference is similarly computed from Eqs. (6) or (7), where the disturbance covariance $W$ is replaced by the covariance of $\boldsymbol{w}_{1}-\boldsymbol{w}_{2}$ and the initial condition on the relative covariance is similarly replaced by the appropriate value. The mean (or expected) value of $\delta \boldsymbol{X}$ is given by

$$
\dot{\bar{\delta} \boldsymbol{X}}=A \overline{\boldsymbol{\delta} \boldsymbol{X}}+B \overline{\boldsymbol{w}}+\Gamma \overline{\boldsymbol{u}}
$$

where $\overline{\boldsymbol{w}}$ and $\overline{\boldsymbol{u}}$ are the mean (relative) disturbance and control and the initial condition is appropriately chosen.

\section{Calculation of Collision Probability}

At any given time the probability that the relative position between the satellites is less than some metric can be computed by integration of the Gaussian probability density function over a region $\mathcal{B}$, which defines the collision and which is centered at the origin of the relative motion coordinate system. The density function defining 


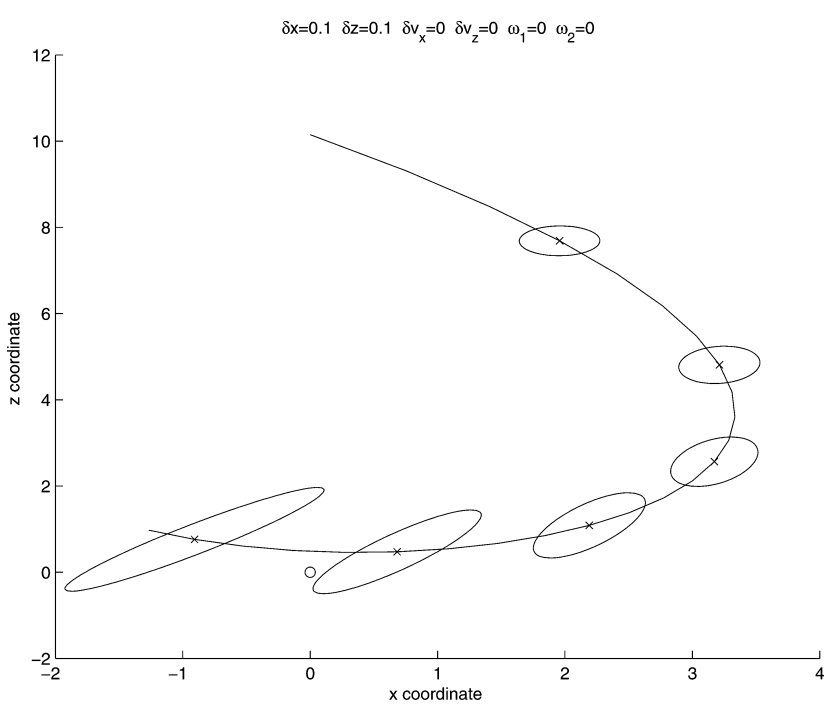

Fig. 3 Evolution of position probability ellipsoid (normalized units) for a near-collision trajectory. Ellipsoids drawn at integer times from 1 to $2 \pi$, where the orbit period is $2 \pi$. Initial position uncertainty is 0.1 units in both $x_{R}$ and $z_{R}$ directions.

the possible relative spacecraft location is centered at $\overline{\boldsymbol{X}}$. Computationally, we find it convenient to reverse these positions, with $\mathcal{B}$ centered at $\overline{\delta \boldsymbol{X}}$, and the probability ellipsoid centered at the origin of coordinates (i.e., the location of the collision object.) Whereas we have shown the ellipsoids in the two-dimensional projected $X-Z$ plane, the relative motion study, and the integration over the density function, must be accomplished in a three-dimensional framework.

For simplicity of presentation, consider a two-dimensional geometry of the relative motion of two satellites as shown in Fig. 3. The initial conditions of the relative motion are chosen intentionally so as to produce a "near" collision. A small initial uncertainty in position is also modeled, and the evolution of the error ellipsoid is also shown on the figure. (Note the contours on the plot are drawn for the $1 \sigma$ ellipsoids.) Because of the secular terms normally present in the $\mathrm{CW}$ equations, the error ellipsoid grows linearly with time and the principle directions of the ellipsoid rotate with the long axis of the ellipse growing in the along-track direction.

To evaluate the collision probability between the two spacecraft, consider the motion in the vicinity of the closest approach. Define a relative coordinate frame with the $x_{R}$ axis in the relative velocity direction at closest approach and the $z_{R}$ axis along the nominal closest approach vector. In the three-dimensional case we define the third $y_{R}$ axis in the conventional right-handed sense. It is also easier to envision the geometry with the error ellipsoid centered at the origin (the position of the reference spacecraft) and the second satellite approaching in the negative $x$ direction. In this frame it is reasonable to consider the local motion to be a constant velocity approach along the nominal $x_{R}$ axis (i.e., we can neglect the curvature of the relative trajectory at this point. This assumption might not be valid if the relative velocity is very small. We will address this point again in the sequel when we find velocity corrections to avoid a potential collision). The nominal closest approach is defined to be at the point where $x_{R}=0$. The $z_{R}$ coordinate is then the nominal closest approach distance. As seen in the preceding figure, the orientation of the principle axes of the probability ellipsoid at the nominal intercept time is generally not aligned with the relative velocity vector. Thus it is generally true that the time for which the probability of collision is a maximum is not at the nominal time of closest approach, but might occur earlier or later depending on the relative size and orientation of the probability ellipsoid.

The probability of a collision at any particular time can be computed by integrating the probability density function over the region that defines the collision. We are generally interested in the probability of collision during an "encounter" rather than the probability that exists at some fixed time. By encounter we mean a trajectory seg-

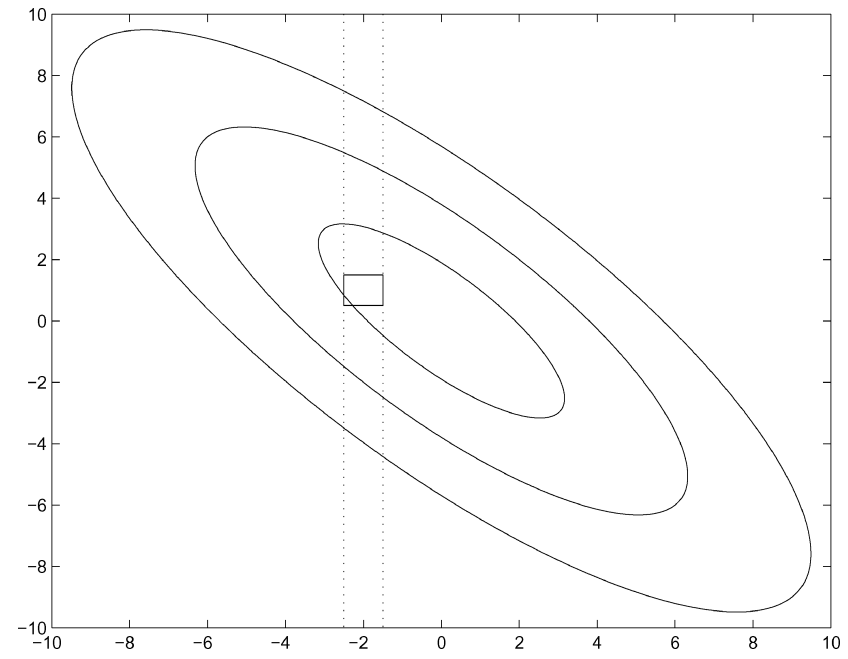

Fig. 4 Equiprobability ellipsoids indicating the probability of collision is determined by integrating the density function across the rectangular volume.

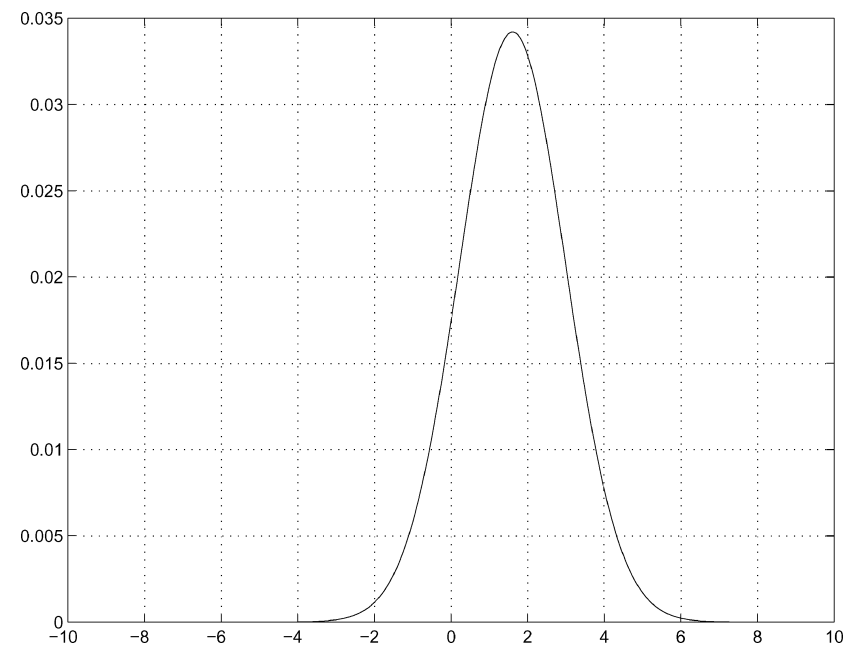

Fig. 5 Probability that the two spacecraft are within the collision region-given as a function of $x$ coordinate for the geometry as shown in Fig. 4.

ment that encompasses the region both before and after the closest approach, during which the probability density function has nonnegligible values. This means that for the collision we need to integrate the density function over a volume which extends sufficiently far in the along-track direction and with cross section equal to the collision cross section. For simplicity here we define the collision region to be a unit square in the two-dimensional region. The collision region is indicated by the square region in Fig. 4. For the case shown in the figure, the "expected" trajectory passes two units to the left of the origin. With the collision region centered at the expected position, the probability as a function of the $x$ coordinate is shown in Fig. 5. For the case shown the maximum probability is relatively small because of the large position uncertainties. In this case also, the peak probability occurs a short time before the predicted nominal closest approach when the collision region is centered in the probability ellipsoid. Neither this maximum probability or the probability at $x_{R}=0$ relate to the actual safety of the encounter. A more important number is the absolute probability of a collision occurring sometime during the pass. For this probability we must integrate the probability density region over the rectangular (in two-dimensions, or the right cylinder in three-dimensions) swath swept out by the collision region during a trajectory pass (see the dotted line in Fig. 4). Although theoretically the integration extends over an infinite distance in the relative motion direction, in practice it is sufficient to restrict the integration region to three to five standard deviations. Note also 


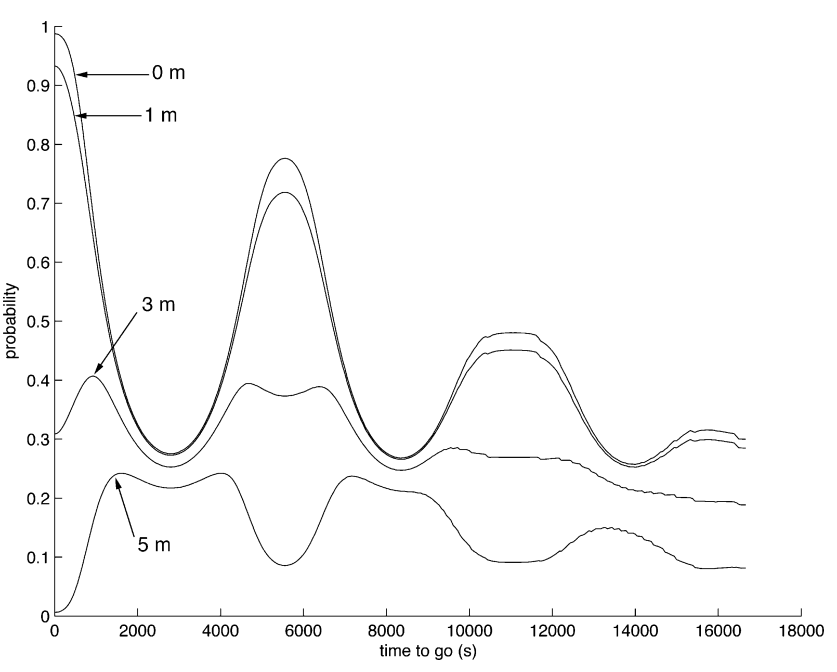

Fig. 6 Probability of collision as a function of time-to-go for trajectories with actual miss distance indicated. The trajectory has $1 \sigma$ uncertainty in initial position uncertainty of $1 \mathrm{~m}$ and a $1 \sigma$ acceleration uncertainty of $10^{-5} \mathrm{~m} / \mathrm{s}^{2}$. The approach angle is zero in all cases.

that in principle if we wish to consider trajectory curvature, we simply need to do the quadrature over the appropriate curved region. For the relative position as shown in the figures, the total probability is still quite small with the probability of a collision computed to be $P_{C}=0.057$. This probability is quite small because the position uncertainty is fairly large compared to the size of the collision region, and the expected trajectory passes well away from the origin. Only when the size of the collision region is comparable to the size of the $3 \sigma$ probability ellipsoid will the collision probability approach one.

The probability density function is calculated forward in time based on the initial uncertainty and any disturbances acting on the spacecraft. A reasonable model is to assume that continuous state estimation maintains the current uncertainty at a more or less constant level. Then integrating from the current time the uncertainty at time of closest approach will be reduced as the time to closest approach draws nearer. We expect in this case that the probability of collision will tend to either zero or one, depending on the predicted closest approach.

Using this model, it is relatively straightforward to calculate probability of collision for the close encounter of two spacecraft in the formation. As an example, we set initial conditions such that the relative motion of two satellites results in a collision or near miss. For this example the collision region is defined (arbitrarily) by a $5-\mathrm{m}$ total breadth, extending $2.5 \mathrm{~m}$ to either side of the origin. The calculated probability of collision as a function of time is shown in Fig. 6 for the case of an initial position uncertainty and a small uncertain disturbing acceleration. A comparable curve with only an initial velocity uncertainty is seen in Fig. 7. The trajectories shown in the figures extend over several orbit periods, but the probability calculation at each time point assumes rectilinear motion in the vicinity of the closest approach and integration of the density function in a $5 \sigma$ range.

An additional complication arises when we consider the angle at which the collision is predicted to occur relative to our along-track and radial coordinate coordinate system. Define the approach angle as the angle between the $X$ axis and the relative velocity vector measured in the counter-clockwise direction. Figures 6 and 7 assume the approach angle is zero (i.e., along the nominal $X$ axis). In the next section the effect of approach angle is considered. The complexity of these curves demonstrate the difficulty that will be associated with collision avoidance within the formation. Because the collision region is defined here to be only marginally larger than the initial covariances, there is relatively small difference in probability between collision and near-collision trajectories, until almost the very end of the trajectory. The numbers are admittedly chosen quite arbitrarily for this example, but given the close proximity of satellites within the nominal formation it is clear that we will need

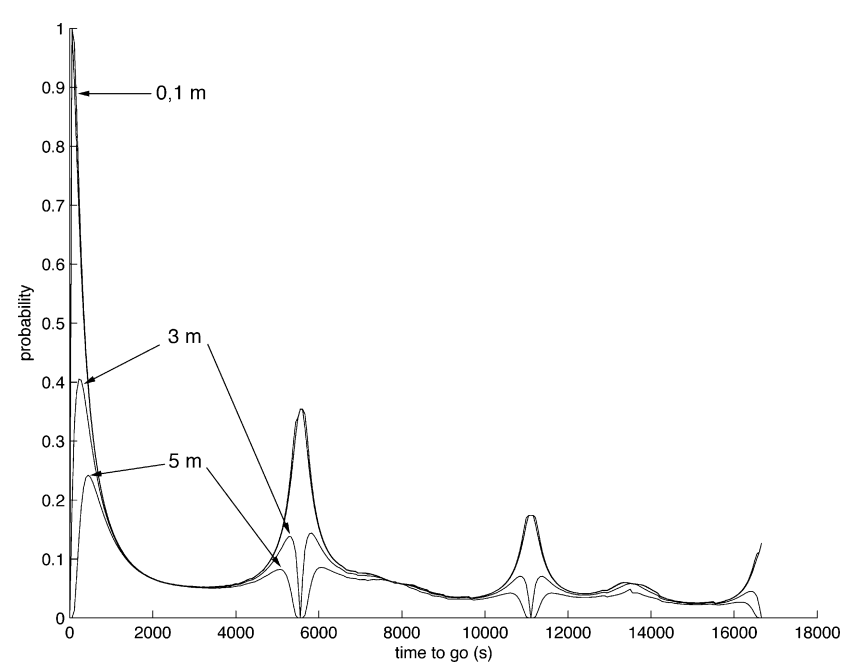

Fig. 7 Probability of collision as a function of time-to-go for trajectories with actual miss distance indicated. The only uncertainty is initial $1 \sigma$ velocity uncertainty of $10^{-2} \mathrm{~m} / \mathrm{s}$ in $X$ and $Z$ directions. The approach angle is zero in all cases.

a very good model of current state and disturbances if we are to adequately predict collision events with significant lead time.

\section{Collision Avoidance}

For satellites in close formation, potential collision with other objects can come from either a foreign object entering the formation or from loss of position control of one of the components of the formation. Given the stochastic nature of the problem, the decision as to when or how to make a velocity correction to avoid a collision is somewhat arbitrary. A decision would be based on the calculated probability that the current states predict a collision is likely to occur in the future and the available $\Delta V$ capability of the spacecraft. The preceding section has shown that the probability of future collision is generally not a monotonic function of time, and further that given (what appear to be) reasonable assumptions on uncertainties in current state and disturbance environment a velocity correction will probably be made before the probability of collision is too high. In this section we examine the problem of computing a velocity correction to create a specific (expected) miss distance between two objects. We use the notation of the preceding section, where we write the relative motion equations with the collision object at the origin of the coordinates, and the motion of the controlled object relative to the origin is described by the linearized model.

Examine first the problem of calculating $\Delta \boldsymbol{V}$ to avoid a collision. Assume that the best estimate of spacecraft states leads to the conclusion that the two vehicles will intersect at some future time $t$. We seek to determine a velocity correction $\Delta \boldsymbol{V}$ to create a miss distance $D$. Because there is an infinite number of ways to compute a velocity correction at any time, we seek the minimum magnitude correction. Assuming the linear model holds, the conditions before any velocity correction are

$$
\left[\begin{array}{l}
\delta \boldsymbol{r}^{(-)} \\
\delta \boldsymbol{V}^{(-)}
\end{array}\right]=\left[\begin{array}{ll}
\phi_{11} & \phi_{12} \\
\phi_{21} & \phi_{22}
\end{array}\right]\left[\begin{array}{l}
\delta \boldsymbol{r}(t) \\
\delta \boldsymbol{V}(t)
\end{array}\right]
$$

where superscript (-) indicates the state at the nominal terminal time $T$ before any velocity correction. Assume for simplicity that the initial state at time $t$ is such that the predicted miss distance $\delta \boldsymbol{r}^{(-)}=0$. If, at time $t$, a velocity correction $\Delta \boldsymbol{V}$ is applied impulsively, then the change of position at the terminal time is

$$
\delta \boldsymbol{r}^{(+)}=\Delta \boldsymbol{R}=\phi_{12} \Delta \boldsymbol{V}
$$

Also the relative velocity at the terminal time will be

$$
\delta V^{(+)}=\delta V^{(-)}+\phi_{22} \Delta V
$$


We pose the problem to find the minimum magnitude of $\Delta V$ at time $t$ such that the terminal position constraint

$$
\left|\Delta \boldsymbol{R}_{\min }\right|^{2}=D^{2}
$$

is satisfied. Because the relative velocity at the final time is nonzero,we must ensure that the miss distance is correctly computed by examining the minimum distance of the relative motion trajectory, which might not occur at the nominal end time. Define $e$ as a unit vector parallel to the nominal final velocity $\delta \boldsymbol{V}^{(+)}$. Assuming the trajectory curvature is negligible in the close vicinity of the terminal time, then the minimum miss is the projection of the distance $\Delta \boldsymbol{R}$ orthogonal to $e$. This can be written succinctly as

$$
\Delta \boldsymbol{R}_{\min }=P \Delta \boldsymbol{R}
$$

where

$$
P=I-\boldsymbol{e} e^{T}
$$

is a symmetric projection matrix. Substituting the preceding and adjoining the constraint, we pose the optimization problem to minimize

$$
J=|\boldsymbol{\Delta} \boldsymbol{V}|^{2}-\lambda\left[\boldsymbol{\Delta} \boldsymbol{V}^{T} \phi_{12}^{T} P \phi_{12} \Delta \boldsymbol{V}-D^{2}\right]
$$

where $\lambda$ is a Lagrange multiplier adjoining the displacement constraint, and we have used the fact that $P^{2}=P$. The solution of this problem is readily interpreted as a minimum norm problem. Differentiating Eq. 18 with respect to the velocity correction vector yields an ordinary eigenvalue problem. The solution for $\lambda$ is the largest eigenvalue of $\phi_{12}^{T} P \phi_{12}$, and the optimal $\Delta V$ is an associated eigenvector with magnitude

$$
|\Delta \boldsymbol{V}|=\sqrt{D^{2} / \lambda_{\max }}
$$

One additional difficulty is in computation of the projection matrix $P$ from Eq. (17). The unit vector $\boldsymbol{e}$ should rightly be interpreted as $\boldsymbol{e}^{(+)}$, which is a unit vector in the direction of the relative velocity after correction. In our numerical approach, we have solved for $\Delta \boldsymbol{V}$ initially using $\boldsymbol{e}$ based on the a priori relative velocity $\delta \boldsymbol{V}^{(-)}$ and then used an iterative contraction algorithm to converge to a solution using $\boldsymbol{e}^{(+)}$. So long as the relative approach velocity is not too small, the effect of this velocity correction is almost a parallel displacement of the relative velocity vector; hence, no iteration on $P$ is actually required. In numerical experiments this holds true for approach velocities as low as $5 \mathrm{~cm} / \mathrm{s}$. At relative approach speeds of $1 \mathrm{~cm} / \mathrm{s}$, the assumption of rectilinear motion is generally not valid, although even here the formal contraction algorithm generates fairly smooth solutions to the optimization problem.

The algorithm equally applies to either the planar (in the orbit plane) or the full three-dimensional encounter geometry. Some typical results for the planar case are shown in Fig. 8, where we have specified a $1-\mathrm{m}$ miss distance. Note that the correction is linear in the desired miss distance $D$, so that the correction for any size maneuver is determined from this solution by multiplying by the desired displacement. Similar shaped curves were computed for the three-dimensional encounter, where we specified (again arbitrarily) a small relative velocity component normal to the orbit plane. These three-dimensional results, which are indistinguishable from the planar curves, are not shown.

One obvious fact evident from the figure is that even where a fixed offset is desired, the best time to apply a velocity correction might be dependent upon the relative approach geometry of the two vehicles and that later might sometimes be better.

\section{A. Effect of Uncertainty Model on Velocity Correction}

In the preceding section we calculated the minimum magnitude velocity correction to achieve a fixed miss distance at the nominal closest approach time. In this case we saw that the size of the correction is not monotonic but can vary considerably within one orbital period. The question arises as to when should a velocity correction be applied given the probabilistic model already discussed. In the

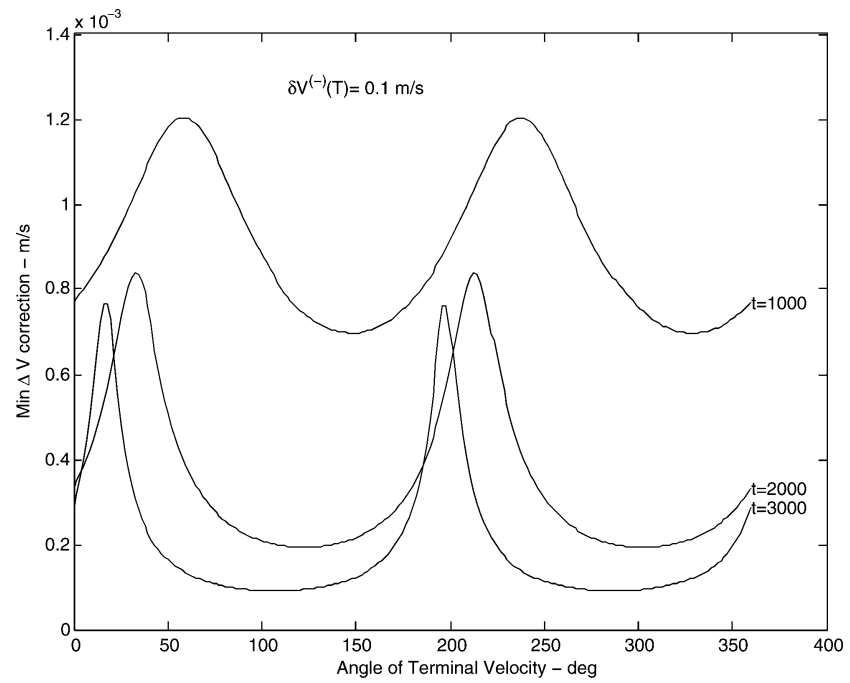

Fig. $8 \Delta V$ correction magnitude required as a function of the direction of the a priori relative velocity at nominal impact for several different values of time-to-go and a miss distance of $1 \mathrm{~m}$. The figure was generated using an a priori relative velocity of $0.1 \mathrm{~m} / \mathrm{s}$; however, the graph is relatively insensitive to this velocity value.

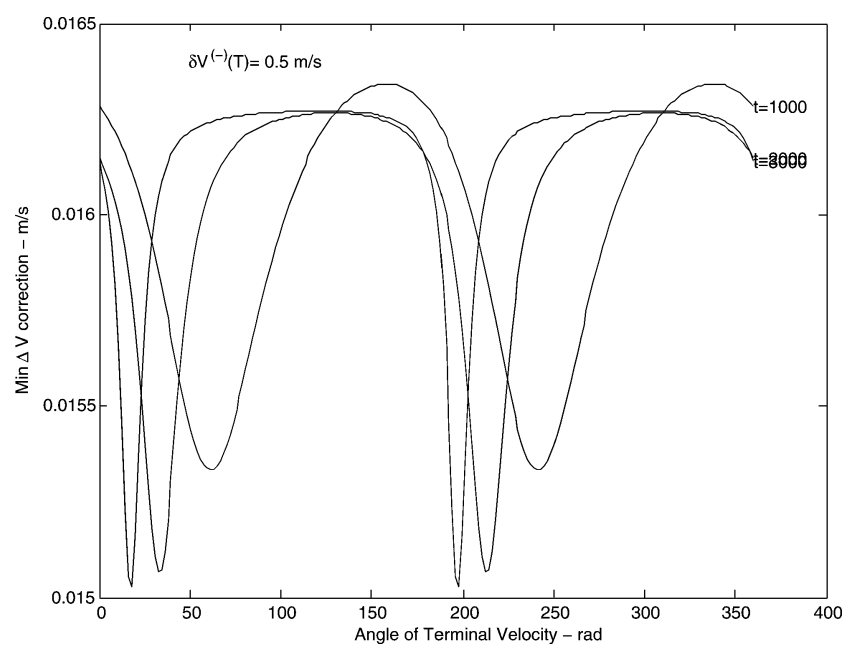

Fig. 9 Minimum $\Delta V$ correction magnitude required as a function of the direction of the a priori relative velocity at nominal closest approach. This correction moves minimum approach distance to the $3 \sigma$ boundary. The a priori relative velocity is $0.5 \mathrm{~m} / \mathrm{s}$. The initial position covariance is $1 \mathrm{~m}$, and the initial velocity covariance is $0.005 \mathrm{~m} / \mathrm{s}$ in each coordinate direction.

actual case uncertain initial conditions, uncertain disturbing accelerations, and even the uncertainty in applying a velocity correction must influence our choice of when to apply a correction.

An intuitive approach would suggest that a velocity correction should be made when the probability of a future collision is high and when the size of the required correction is small (but definitely when the size of correction is within the available control capability of the satellite). To examine the behavior of the required velocity correction, we compute a velocity correction strategy based on the methodology of the preceding section, such that the predicted miss distance is moved to the $3 \sigma$ boundary of the covariance ellipse. The $\Delta \boldsymbol{V}$ required in an example case is shown in Fig. 9. This case is for a fairly high relative velocity $(0.50 \mathrm{~m} / \mathrm{s})$ at the terminal time. $\Delta V \mathrm{~s}$ are computed for times up to $3000 \mathrm{~s}$, which is slightly more than half an orbital period. Because this model assumes linear motion in the vicinity of the origin, the range of initial velocities for which we can compute valid results is dependent upon the size of the initial uncertainty (and thus the distance $\Delta R_{\min }$, which is proportional to the final position covariance). We have computed cases with the final relative speed as low as $2 \mathrm{~cm} / \mathrm{s}$. But with this final speed the 
initial covariances must be reduced, or the size of the $3 \sigma$ ellipsoid would be comparable to the distance traveled for the times in our example. As a general rule, we have found this algorithm gives valuable results when the curvature of the nominal trajectory near the endpoint is small relative to the curvature of the $3 \sigma$ ellipsoid, which we are avoiding.

Note that in the case shown in the figure the velocity correction magnitude varies only slightly with time of correction. This can be explained by noting from our preceding results that the uncertainty as a result of initial conditions grows linearly with time, while the size of the correction varies inversely with the magnitude of the $\phi_{12}$ eigenvalue, which similarly grows linearly with time. The result is that the required $\Delta \boldsymbol{V}$ magnitude in this model is relatively constant. Thus interestingly, the time of correction is not important in determining the size of the required correction magnitude. We have not explicitly included the effect of disturbing acceleration nor uncertainty in magnitude and direction of the velocity correction. Both of these effects can be nonnegligible in a realistic case and can affect these conclusions.

\section{Conclusions}

In this paper we present a method for determining collision probabilities based on best state and disturbance information and on determining a minimal velocity correction to avoid a future collision. Numerically however we find that this simplistic strategic can be difficult to implement if precise control is required. Because of the periodic nature of some of the dominant covariance terms, the probability of collision is far from monotonic, and in addition the probability of collision of collision can approach one, only when the time to collision is a small fraction of an orbit. Our conclusion is that accurate state estimation is essential to an efficient collisionavoidance algorithm and that the specific correction capability of the satellite will determine when to specify a velocity correction.

An optimal projection method, which allows the minimum norm velocity correction to be applied at any time, is determined. This correction should be applied at a time when the correction is small enough to be within the capability of the satellite, and the probability of future collision is sufficiently high enough to justify the expenditure of this energy. The "best" time then for a correction is probably going to be decided by a number of factors not considered in this paper. Additional criteria such as missed detection and false alarm can be amenable to a Bayesian approach.

Future work that merges these results with a better model of the navigation errors and the disturbance environment should allow us to better formulate a strategy for optimal correction maneuvers. Additionally, we have only examined the velocity correction to avoid a collision with a single other spacecraft. Further work is required to include the problem of maintaining (or returning to) position in the formation.

\section{Acknowledgement}

This research was supported by NASA Goddard Space Flight Center Cooperative Agreement NCC5-728. Any opinions, findings, and conclusions or recommendations expressed in this material are those of the authors and do not necessarily reflect the views of NASA.

\section{References}

${ }^{1}$ Carpenter, J. R., Leitner, J. A., Folta, D. C., and Burns, R., "Benchmark Problems for Spacecraft Formation Flying Missions," AIAA Paper 20035364, Aug. 2003.

${ }^{2}$ Carpenter, J. R., "Decentralized Control of Satellite Formations," International Journal of Robust and Non-Linear Control, Vol. 12, Nos. 2-3, 2002, pp. 141-161.

${ }^{3}$ Sabol, C., Burns, R., and McLaughlin, C., "Satellite Formation Flying Design and Evolution," Journal of Spacecraft and Rockets, Vol. 38, No. 2, 2003. pp. 233-237.

${ }^{4}$ Chan, K., "Formation Flying: Collision Assessment, Pre-Emptive Maneuvers and Safe Haven Parking," Proceedings of the 2003 Flight Mechanis Symposium, NASA Goddard Space Flight Center, Greenbelt, MD, Oct. 2003.

${ }^{5}$ Alfriend, K. T., Akella, M. R., Frisbee, J., Foster, J. L., Lee, D., and Wilkins, M., "Probability of Collision Analysis," Space Debris, Vol. 1, No. 1, 2000. pp. 21-35.

${ }^{6}$ Patera, R. P., "Satellite Collision Probability for Nonlinear Relative Motion," Journal of Guidance, Control, and Dynamics, Vol. 26, No. 5, 2003, pp. 728-733.

${ }^{7}$ Patera, R. P., and Peterson, G., "Space Vehicle Maneuver Method to Lower Collision Risk to an Acceptable Level." Journal of Guidance, Control, and Dynamics, Vol. 26, No. 2, 2001, pp. 270-278.

${ }^{8}$ Clohessy, W. H., and Wiltshire, R. S., "Terminal Guidance System for Satellite Rendezvous," Journal of the Aerospace Sciences, Vol. 27, No. 5, 1960, 653-658.

${ }^{9}$ Stern, R., "Interplanetary Midcourse Guidance Analysis," Ph.D. Dissertation, Massachusetts Inst. of Technology, Dept. Aeronautics and Astronautics, June 1963.

${ }^{10}$ Bryson, A. E., Jr., and Ho, Y. C., Applied Optimal Control, Taylor and Francis, New York, 1975, Chap. 10.

${ }^{11}$ Williams, T., Register, J., and Slater, G., "Formation-Keeping Maneuver Dispersions and Safe Mode Insertion for Satellite Formation Flight," AIAA Paper 2004-4734, Aug. 2004. 